\title{
DISRUPTIVE SELECTION WITH FIXED OPTIMA
}

\author{
D. O. F. SKIBINSKI* and J. M. THODAY \\ Department of Genetics, University of Cambridge, Cambridge, U.K.
}

Received 20.v.78

\section{SUMMARY}

The effect of artificial disruptive selection using fixed optima instead of selecting extreme phenotypes was investigated with the character sternopleural chaeta number in Drosophila melanogaster. The prediction that this selection will lead to a bimodal phenotypic distribution is not supported by the results obtained.

\section{INTRODUCTION}

Selection on a continuous character can be classified into three main types, directional, stabilising, and disruptive (Mather, 1953). Disruptive selection occurs when two or more different phenotypes are favoured simultaneously by selection within a single population.

Mather (1955) suggested that disruptive selection with two optimal phenotypes could result in a dimorphism with conversion of a unimodal to bimodal phenotypic distribution. Bimodality could be achieved either by the formation of two reproductively isolated populations or by the creation of a genetic or environmental mechanism to switch ontogenetic development into one of two alternative pathways.

Laboratory experiments, mainly with Drosophila have shown that artificial disruptive selection can produce both switch-like genetic polymorphisms consisting of groups of closely linked loci (Thoday, 1972, for review) and phenotypic dimorphisms (Scharloo, 1970a,b).

This paper describes an investigation of the effect of disruptive selection using fixed selection optima as an alternative to the usual method of selecting extreme phenotypes. It is expected that this selection will result in a phenotypic dimorphism with the two modes coinciding with the optima, or, less specifically, to an increase in frequency of individuals at the selected optima. Such predictions can be tested more easily with fixed optima than with extreme selection where the phenotypic values of selected parents change from generation to generation.

In a panmictic population segregation of two alleles at a single locus can provide a genetic switch if one allele is dominant. Although large dominance deviation values have been found associated with genetic polymorphisms produced by artificial disruptive selection (Wolstenholme and Thoday, 1963; Scharloo, 1970a) these past experiments were not concerned with dominance modification. By contrast, the experiments described here were designed to cause specific changes in the direction of dominance.

* Present address: Department of Genetics, University College of Swansea, Singleton Park, Swansea, U.K. 


\section{Materials and methods}

\section{(i) Materials}

A base population of Drosophila melanogaster was derived from a single inseminated female captured in an orchard at Eversden near Cambridge in October 1970. The character scored was the sum of left and right sternopleural chaetae. Cultures were reared in milk bottles with agar oatmeal medium, and kept in the dark at $25 \mp 1^{\circ} \mathrm{C}$.

\section{(ii) The quasi-random mating system}

Each disruptive selection line began with four bottles each with four unselected virgin females and four males. Progeny from these bottles was generation $G_{0}$. The chosen optima were 17-18 chaetae (low) and 22-23 chaetae (high). From each generation eight high and eight low flies of each sex were selected as parents from the progeny and then mated in quasi-random fashion (Gibson and Thoday, 1963). The 32 parents were distributed among four culture bottles as follows:

\begin{tabular}{|c|c|c|c|}
\hline \multicolumn{4}{|c|}{ CULture } \\
\hline $\begin{array}{c}\text { non-hybrid } \\
\text { HH }\end{array}$ & $\begin{array}{l}\text { hybrid } \\
\text { HL }\end{array}$ & $\begin{array}{l}\text { hybrid } \\
\text { LH }\end{array}$ & $\begin{array}{l}\text { non-hybrid } \\
\text { LL }\end{array}$ \\
\hline $\begin{array}{l}\text { high }(22-23) \text { 우우 } \\
4 \text { high }(22-23) \sigma^{0} \sigma\end{array}$ & $\begin{array}{l}4 \text { high }(22-23) \text { 웅 } \\
+4 \text { low }(17-18) 0^{\circ} \sigma^{2}\end{array}$ & $\begin{array}{l}4 \text { low }(17-18) \text { 우 } \\
+4 \text { high }(22-23) \sigma^{3} \sigma^{3}\end{array}$ & $\begin{array}{l}4 \text { low }(17-18) \text { 우 } \\
\quad+4 \text { low }(17-18) \sigma^{3} 0\end{array}$ \\
\hline
\end{tabular}

Each generation, males and virgin females were collected, and 20 flies of each sex scored from each culture bottle making a total of 160 scored overall. When choosing parents, priority was given first to optimal flies, and then to flies either side of the optima if insufficient optimal flies were present in the sample scored.

\section{(iii) Experimental designs}

There were three experimental designs each having different modifications to the quasi-random mating system. In all experiments $\mathrm{HH}$ and LL cultures were not allowed to contribute low and high parents respectively; this was to facilitate divergence between $\mathrm{HH}$ and $\mathrm{LL}$ means. Additional modifications were as follows.

In Experiment I (called High Low Recipient, abbreviated HLR) there were no further modifications. The high parents were selected at random from the pooled optimal progeny of the $\mathrm{HH}, \mathrm{HL}$ and $\mathrm{LH}$ cultures: low parents were selected from the HL, LH and LL cultures. Hybrid cultures, HL and LH, could therefore contribute to both high and low parental groups. This experiment was replicated twice.

In Experiment II (High Recipient, HR) high parents were selected from the progeny of the $\mathrm{HH}, \mathrm{HL}$ and $\mathrm{LH}$ cultures: low parents were selected from the LL culture alone. Hybrid cultures contributed only to the high parental group. This experiment was replicated four times.

In Experiment III (Low Recipient, LR) low parents were selected from the progeny of the HL, LH and LL cultures: high parents were selected from the $\mathrm{HH}$ culture alone. Hybrid cultures contributed only to the low parental group. This experiment was also replicated four times. 
There were therefore 10 disruptive selection lines in all. Selection was carried on for 30 generations in each.

\section{(iv) Rationale of experimental designs}

The HR and LR experiments were designed to lead to increased dominance of high and low chaeta factors respectively. The reasoning is as follows. It was assumed, correctly, that the hybrid culture means and distributions would, as in past disruptive selection experiments, quickly become intermediate between $\mathrm{HH}$ and LL. A large proportion of hybrid individuals would probably be heterozygous for high and low chaeta factors selected mainly as homozygotes in the non-hybrid cultures. In the HR lines modifiers causing greater dominance of high factors would be at a selective advantage and should increase in frequency. Similarly, modifiers causing greater dominance of low chaeta factors should increase in frequency in the LR lines. The difference between HR and LR should be reflected by changes in the position of the hybrid culture means which should move closer to the HH mean in HR and the LL mean in LR. In HLR increased dominance of both high and low chaeta factors would be selected and the position of hybrid culture means would be expected not to change as much as in $H R$ and $L R$. HLR can therefore be regarded as control. An increase in average high (low) dominance in HR (LR) could also be achieved by an increase in frequency of those among the high (low) chaeta factors that showed the greatest dominance.

\section{RESULTS}

In the base population, mean, variance and heritability were estimated to be $20 \cdot 0 \pm 0 \cdot 15,4 \cdot 6$, and $0 \cdot 6$ respectively. The optima which were roughly one standard deviation above and below the mean were placed close enough together to ensure that hybrid progeny would always contribute to the high and low parental groups thus preventing isolation of $\mathrm{HH}$ and LL cultures. In fact, hybrid cultures contributed approximately 50 per cent of the high (in HLR and HR) and low (in HLR and LR) parents throughout the course of selection.

\section{(i) Chaeta distributions}

Fig. I shows chaeta distributions for each line for $\mathrm{G}_{26}$ to $\mathrm{G}_{30}$ (a combined total of $5 \times 160=800$ individuals) and for the base population (1600 individuals). Hybrid distributions are intermediate between $\mathrm{HH}$ and LL but overlap of component culture distributions is considerable. There is no evidence of bimodality. There is no value in presenting distributions for individual generations; large sampling variation hinders interpretation.

As an aid to assessment of trends obscured by fluctuations between generations, the responses have been divided into three "periods" each of 10 generations. The first period (P1) includes $G_{1}$ to $G_{10}, P 2$ includes $G_{11}$ to $G_{20}$, and $P 3$ includes $G_{21}$ to $G_{30}$. The 10 values of a variable are used to provide a representative value for the period. Table 1 gives numbers of individuals with optimal phenotypes (high plus low) out of the 1600 scored each period. Seven lines and the grand totals show a decrease from $\mathrm{Pl}$ to $\mathrm{P} 3$, and in $\mathrm{P} 3$ seven lines have a lower number than in the base 


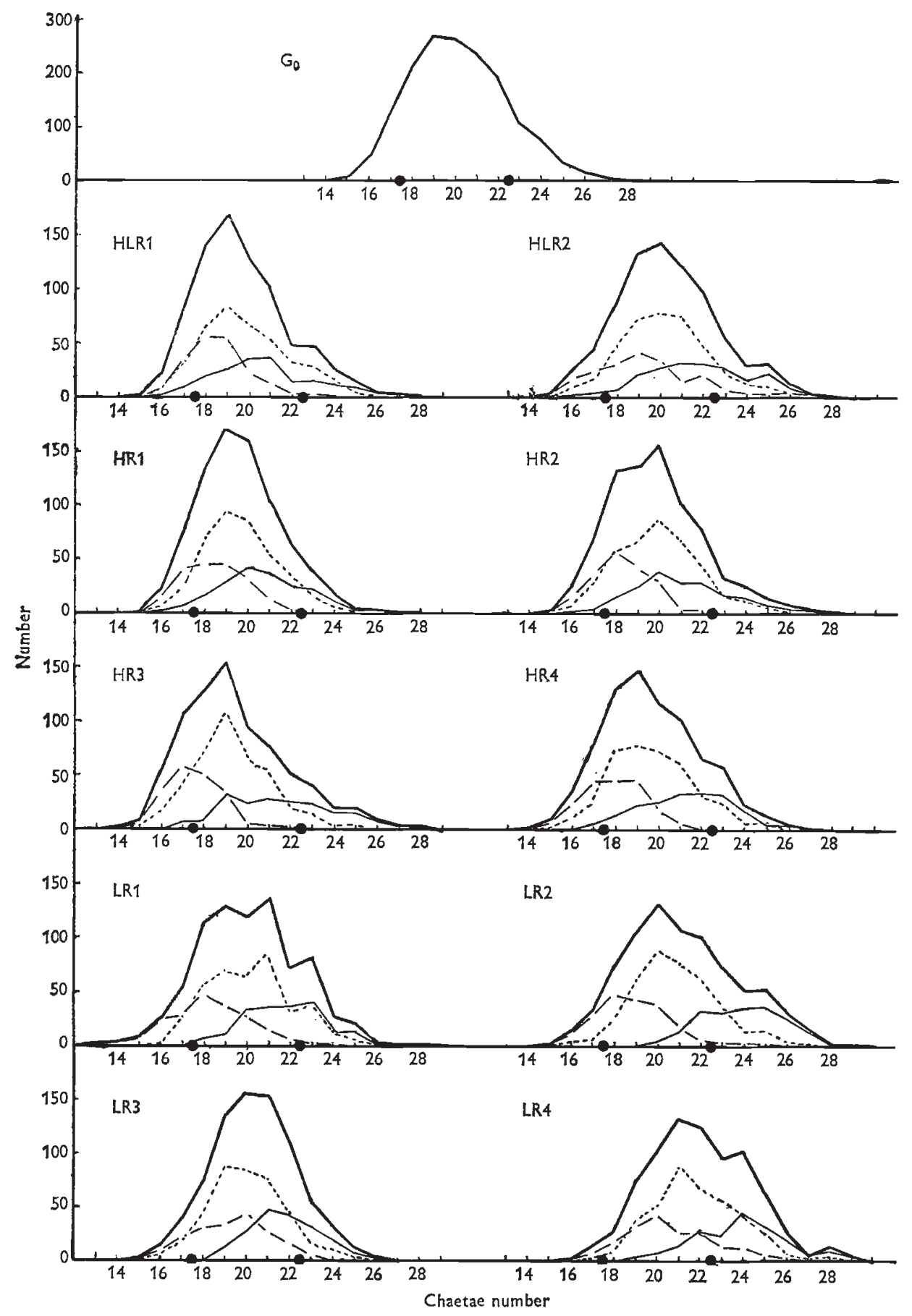

Frg. 1.-Chaetae distributions for $\mathrm{G}_{26}$ to $\mathrm{G}_{30}$ combined. $---=\mathrm{LL},--\mathrm{HH}$, ....- Hybrid $(\mathrm{HL}+\mathrm{LH}), \quad-=$ total; 0 mean optimal chaetae numbers. 
population. Therefore selection has not caused any significant increase in the proportion of optimal individuals.

TABLE 1

Numbers of optimal flies

$\begin{array}{llll}\text { Line } & \text { P1 } & \text { P2 } & \text { P3 } \\ \text { HLR1 } & 651 & 620 & 615 \\ \text { HLR2 } & 653 & 608 & 619 \\ \text { HR1 } & 595 & 601 & 597 \\ \text { HR2 } & 717 & 626 & 654 \\ \text { HR3 } & 650 & 633 & 696 \\ \text { HR4 } & 628 & 703 & 673 \\ \text { LR1 } & 650 & 599 & 608 \\ \text { LR2 } & 613 & 593 & 610 \\ \text { LR3 } & 623 & 662 & 619 \\ \text { LR4 } & 659 & 587 & 546 \\ \text { Totals } & \overline{6439} & 6232 & \overline{6237}\end{array}$

Number in $G_{0}=628$ out of 1600

(ii) Culture means

Period mean values for the four cultures and for high and low parents are given in fig. 2. Hybrid means are intermediate, and parental means are closer than non-hybrid progeny means to the mean optimal values of 17.5 and 22.5 . For each period in each line, values for the variables divergence (HH-LL), (HL-LH), and heterosis have been calculated. Heterosis (Falconer, 1960) gives an indication of the direction and amount of dominance by measuring the position of the hybrid in relation to the $\mathrm{HH}$ and $\mathrm{LL}$ mean. It is defined:

$$
\text { Heterosis }=((\bar{x} \mathrm{HL}+\bar{x} \mathrm{LH})-(\bar{x} \mathrm{HH}+\bar{x} \mathrm{LL})) / 2,
$$

where $\bar{x}$ is culture period mean. For each variable a two-way analysis of variance has been made with 10 (lines) $\times 3$ (periods) $-1=29$ degrees of freedom, using replicates and replicates $\times$ periods as error variance. Mean values for the three treatments (HR, LR, and HLR) are given in table 2; probability levels for significant comparisons (always involving LR) are indicated between the tabulated values. Also given are values for crosses between high and low optimal flies in the base population $\left(G_{1}\right)$. In the

TABLE 2

Heterosis, $H L-L H$, and divergence for three experimental designs

\begin{tabular}{lrrrrrc} 
& HR & HRvsLR & LR & LRvsHLR & \multicolumn{1}{c}{ HLR } & $\mathrm{G}_{1}$ \\
Heterosis & +0.01 & $\mathrm{P}<0.01$ & -0.34 & $\mathrm{P}<0.01$ & -0.01 & $-0.34 \pm 0.16$ \\
HL-LH & -0.28 & $\mathrm{P}<0.05$ & +0.07 & n.s. & -0.03 & $+0.29 \pm 0.28$ \\
$\begin{array}{l}\text { Divergence } \\
\begin{array}{l}\text { Relative } \\
\text { heterosis }\end{array}\end{array}$ & 2.56 & $\mathrm{P}<0.05$ & 3.35 & $\mathrm{P}<0.05$ & 2.52 & - \\
$\begin{array}{c}\text { Relative } \\
\quad \text { heterosis } \\
\text { (log trans.) }\end{array}$ & +0.78 & - & -20.30 & - & -0.79 & - \\
$\quad$ & +4.13 & - & -17.55 & - & +2.51 & - \\
$\quad$ 42/3-D & & & & & &
\end{tabular}



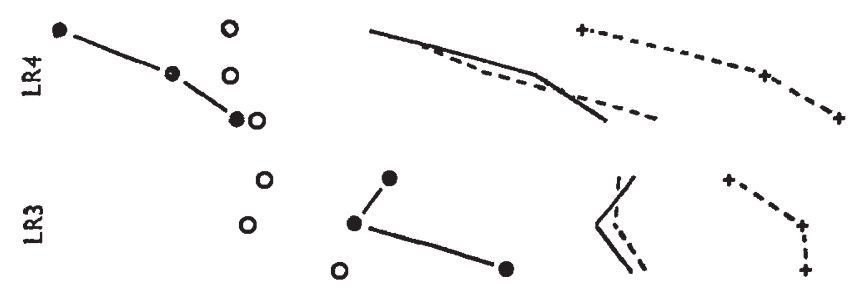

$\circ$
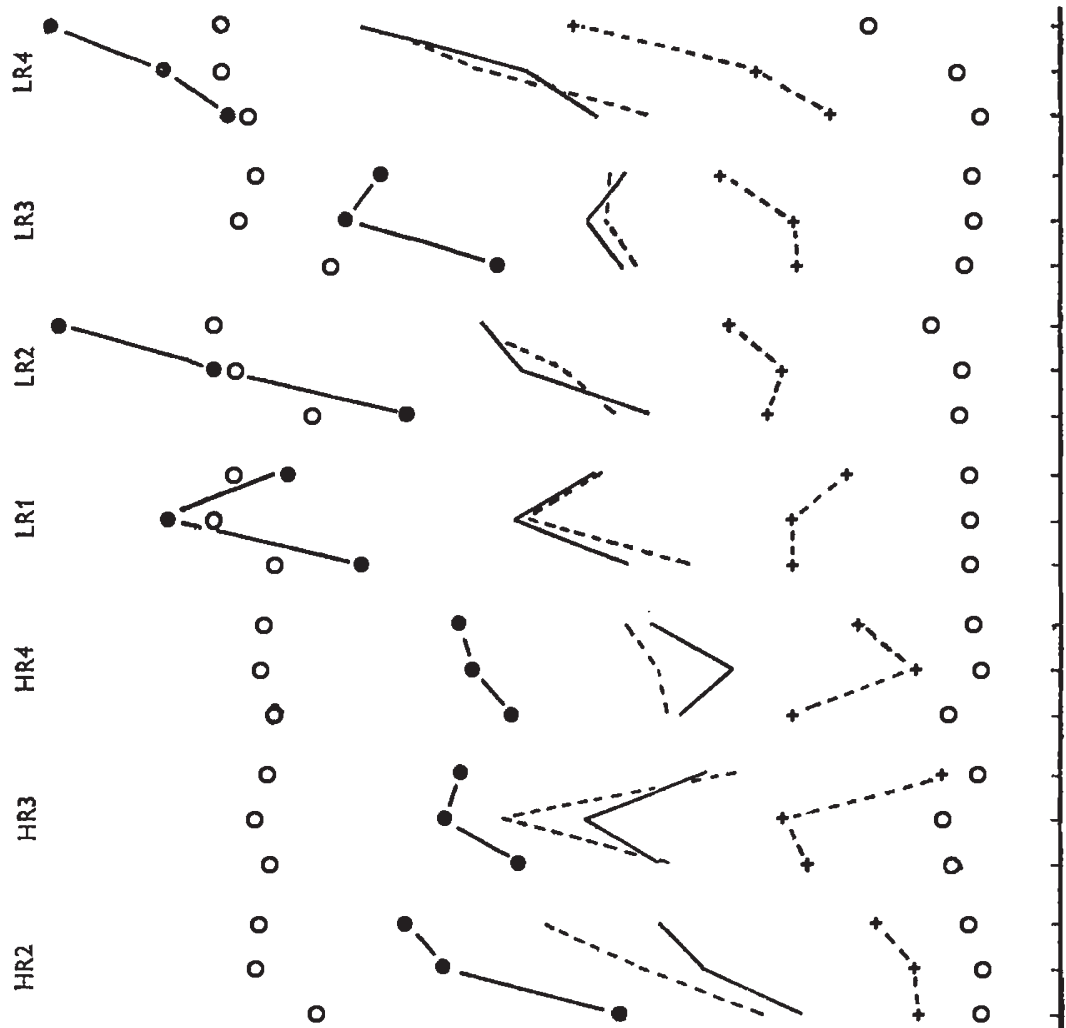

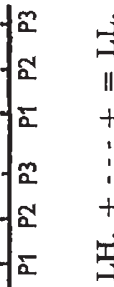
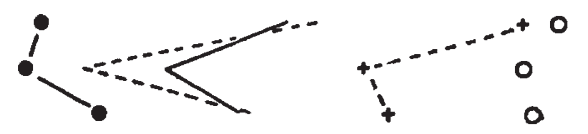

analysis, periods $\times$ treatments components were non-significant suggesting the absence of consistent trends; differences between treatments evolved rapidly.

For heterosis, the more negative value for $L R$ than $H R$ is as predicted. HLR, the control, is however not intermediate. The negative value for the base population suggests that change has occurred mainly in HLR and 
HR. For divergence, HR and HLR are similar; LR has a greater value than, and is significantly different from, both.

A striking feature of the results concerns the responses of LR2 HH and LR4 HH. In both, mean chaeta number increased to $23 \cdot 7 \pm 0.3$ in P3 although parents were always close to the mean optimal value of 22.5 (fig. 2). In the isolated non-hybrid cultures selection has, in general, been more effective in the high than low direction. This appears to be the major cause of the higher divergence and culture mean values in LR. It is possible, therefore, that the difference in heterosis between $H R$ and $L R$ is a scale effect attributable to the responses of the isolated non-hybrid culture. Differences in divergence can be corrected by calculating: Relative Heterosis $=($ Heterosis $\times 200) /$ divergence, and a logarithmic transformation is appropriate for correcting differences in mean for this character. Transformed and untransformed values of Relative Heterosis are given in table 2; the differences among experimental designs still remain.

The lower values of HL-LH as compared with the base population are consistent with the selection of a paternal effect which is greater in magnitude in HR than LR. However, by this hypothesis a more negative value is expected for HLR than for either HR or LR; this is not observed. The pattern of differences for HL-LH is the same in both sexes; if a paternal effect is involved this would implicate the paternal cytoplasm rather than the $\mathrm{Y}$ chromosome.

\section{Discussion}

Disruptive selection for 30 generations has failed to cause the predicted modification of the chaeta number distribution. This contrasts with results of both artificial directional selection, where it is notoriously difficult not to obtain a response (Lewontin, 1974), and artificial stabilising selection where predicted decreases in genetic and environmental variance have been observed repeatedly (Thoday, 1959; Prout, 1962; Scharloo, 1964; Gibson and Bradley, 1974). There are two principal interpretations of this negative result. First, the prediction may not be well founded. Intuitively it may seem reasonable to expect disruptive selection to convert a unimodal to bimodal distribution; there is, however, no general genetic theory that gives this prediction. An important unsolved problem is to ensure the evolution of a mechanism that causes a reduction in the frequency of segregation of offspring with intermodal phenotypes. A second interpretation is that the experimental design was particularly unfavourable to produce the desired end result. Some extracted second and third chromosomes showed large differences in effect on chaeta number in relation to the distance between optima (Skibinski, 1976) so it is possible that genetic polymorphisms of large effect were produced but obscured by environmental variation; in practice it is difficult to detect segregation at even single loci unless heritability is high (Thoday and Thompson, 1976). In retrospect it might have been wise to place the optima further apart initially as there was never any danger of isolation of high and low parental groups. Successful production of bimodality by disruptive selection is probably increased when use is made of a truncated character such as wing vein length (Scharloo, Hoogmoed, and Ter Kuile, 1967), where the optima correspond with the phenotypic limits to selection.

The difference in heterosis between $H R$ and LR was established in the 
early stages of selection. If dominance modification was involved, it is therefore more likely that this was achieved through changes in allele frequencies at chaeta loci already showing some degree of dominance rather than by selection for modifiers of dominance deviation values. Dominance relationships of major locus alleles segregating within single populations can be altered by artificial selection (Ohh and Sheldon, 1970) so the latter alternative is conceivable. There is no reason to doubt the existence of dominance modifiers of polygenic alleles especially as there is evidence that some polygenes are iso-alleles of major loci (Thompson, 1975).

The hypothesis that the heterosis difference between HR and LR is an artifact attributable to the responses of the isolated non-hybrid cultures is not supported when corrections are made for mean and divergence. At the same time the finding of similar heterosis values in HLR and HR is not in line with predictions and is difficult to interpret.

The responses of LR2 $\mathrm{HH}$ and LR4 $\mathrm{HH}$ in the face of artificial selection are most likely to have resulted from strong natural selection; it is significant that lethal alleles were identified at high frequencies in many cultures (Skibinski, 1976). Unfortunately these findings only add uncertainty to the interpretation of the heterosis difference.

In conclusion, it appears that although disruptive selection might have altered genetic architecture in a predictable way it has failed to produce phenotypic dimorphisms with genetic switches showing dominance of either high or low chaetae alleles. In the main, the results do not provide good experimental support for the expectations outlined in the Introduction.

While criticism is often made of attempts to draw general theoretical conclusions from the results of such experiments it must be remembered that artificial selection frequently provides the only method of demonstrating that selection has those effects that we believe it to.

Acknowledgments.-We thank Drs D. S. Angus, K. J. R. Edwards, and J. N. Thompson for advice and criticism. This work was done while D. O. F. S. was in receipt of an S.R.C. studentship.

\section{REFERENCES}

FALCONER, D. S. 1960. An Introduction to Quantitative Genetics. Oliver and Boyd, Edinburgh. GIBSON, J. B., AND BRADLEY, B. P. 1974. Stabilising selection in constant and fluctuating environments. Heredity, 33, 293-302.

GIBSON, J. B., AND THODAY, J. M. 1963. Effects of disruptive selection. VIII. Imposed quasi-random mating. Heredity, 18, 513-524.

LEwontin, R. C. 1974. The Genetic Basis of Evolutionary Change. Columbia Univ. Press.

MATHER, K. 1953. The genetical structure of populations. Symp. Soc. Exp. Biol., 7, 66-95.

MATHER, K. 1955. Polymorphism as an outcome of disruptive selection. Evolution, 9, 52-61. OHH, B. K., AND SHELDON, B. L. 1970. Selection for dominance of Hairy-wing (Hw) in Drosophila melanogaster. I. Dominance at different levels of phenotype. Genetics, 66, 517-540.

Prout, T. 1962. The effects of stabilising selection on the time of development in Drosophila melanogaster. Genet. Res., 3, 364-382.

scharloo, w. 1964. The effect of disruptive and stabilising selection on the expression of a cubitus interruptus mutant in Drosophila. Genetics, 50, 553-562.

scharloo, w. 1970a. Stabilising and disruptive selection on a mutant character in Drosophila. II. Polymorphism caused by a genetical switch mechanism. Genetics, 65, 681-691.

SCHARLOO, w. 1970b. Stabilising and disruptive selection on a mutant character in Drosophila. III. Polymorphism caused by a developmental switch mechanism. Genetics, 65, 693-705. 
SCHARLOO, W., HOOGMOED, M. S., AND TER KUILE, A. 1967. Stabilising and disruptive selection on a mutant character in Drosophila. I. The phenotypic variance and its components. Genetics, 56, 709-726.

SKIBINSKI, D. O. F. 1976. Disruptive Selection with Fixed Optima. Unpublished Doctoral Dissertation, Cambridge University.

тнорау, J. м. 1959. Effects of disruptive selection. I. Genetic flexibility. Heredity, 13, 187-203.

THoday, J. M. 1972. Disruptive selection. Proc. Roy. Soc. B., 182, 109-143.

THODAY, J. M., AND THOMPSON, J. N. 1976. The number of segregating genes implied by continuous variation. Genetica, 46, 335-344.

THOMPSON, J. N. 1975. Quantitative variation and gene number. Nature, 258, 665-668.

WOLSTENholme, D. R. AND THODAy, J. M. 1963. Effects of Disruptive Selection VII. A third chromosome polymorphism. Heredity, 18, 413-431. 